\title{
Numerically Investigating the Effects of Cross Links in Scaled Microchannel Heat Sinks
}

\author{
M. Dang, I. Hassan ${ }^{1}$, and S. I. Kim \\ Department of Mechanical and Industrial Engineering \\ Concordia University \\ Montreal, Quebec, Canada
}

\begin{abstract}
Thermal management for high performance of miniaturized electronic devices using microchannel heat sinks has recently become of interest to researchers and industry. Obtaining heat sink designs with uniform flow distribution is strongly desired. A number of experimental studies have been conducted to seek appropriate designs for microchannel heat sinks. However, pursuing this goal experimentally can be an expensive endeavor. The present work investigates the effect of cross-links on adiabatic two-phase flow in an array of parallel channels. It is carried out using the three-dimensional mixture model from the computational fluid dynamics (CFD) software, Fluent 6.3. A straight channel and two cross-linked channel models were simulated. The cross-links were located at $1 / 3$ and $2 / 3$ 's of the channel length, their width varied by one and two times the channel width. All test models had 45 parallel rectangular channels, with a hydraulic diameter of 1.59 $\mathrm{mm}$. The results showed that the trend of flow distribution agrees with experimental results. A new design, with cross-links incorporated, was proposed and the results showed a significant improvement, up to $55 \%$, on flow distribution, compared to the standard straight channel configuration without a penalty in the pressure drop. The effect of cross-links on flow distribution, flow structure, and pressure drop was also documented.
\end{abstract}

NOMENCLATURE

$\boldsymbol{Q}_{\boldsymbol{G}} \quad$ Volumetric of air $\left(\mathrm{m}^{3} / \mathrm{s}\right)$

$\boldsymbol{Q}_{\boldsymbol{m}} \quad$ Volumetric of mixture $\left(\mathrm{m}^{3} / \mathrm{s}\right)$

$\beta_{G} \quad$ Volume fraction of air

$m_{G} \quad$ Mass flow rate of air, $\mathrm{kg} / \mathrm{s}$

\footnotetext{
${ }^{1}$ Corresponding author - Email: ibrahimH@alcor.concordia.ca
}

\author{
$m_{L} \quad$ Mass flow rate of liquid, $\mathrm{kg} / \mathrm{s}$ \\ $\mathrm{j}_{\mathrm{G}} \quad$ Superficial gas velocity, $\mathrm{m} / \mathrm{s}$ \\ $\mathrm{j}_{\mathrm{L}} \quad$ Superficial liquid velocity, $\mathrm{m} / \mathrm{s}$ \\ x Flow quality, $x=\frac{\dot{m}_{G}}{\dot{m}_{G}+\dot{m}_{L}}$ \\ $\mu \quad$ Dynamic viscosity, N.s $/ \mathrm{m}^{2}$ \\ $\mathrm{V} \quad$ Velocity, $\mathrm{m} / \mathrm{s}$ \\ A Cross-sectional area of channel, $\mathrm{m}^{2}$ \\ $\mathrm{P} \quad$ Channel wetted perimeter, $\mathrm{m}$ \\ $\mathrm{D}_{\mathrm{h}} \quad$ Hydraulic diameter, $\left(\frac{4 A}{P}\right)$ \\ $\mathrm{L} \quad$ Length of channels \\ Re Reynolds number, $\left(\frac{\rho V \mathrm{D}_{\mathrm{h}}}{\mu}\right)$ \\ $f \quad$ Friction faction \\ Greek \\ $\rho \quad$ Density, $\mathrm{kg} / \mathrm{m}^{3}$ \\ Subscripts \\ G Gas, air \\ $L \quad$ Liquid, water \\ $m \quad$ Mixture \\ TP Two-phase

\section{INTRODUCTION}

In the past decades, heat sinks have become one of the emerging cooling approaches to improve thermal management for high performance of miniaturized electronics devices. Since 
the pioneering work of Tuckerman and Pease (1981), many various studies have been conducted to investigate heat transfer characteristics in microchannel heat sinks. These studies included flow distribution and flow pattern investigations involving microchannel heat sinks. A large number of works have been therefore experimentally conducted to investigate such studies. However, mal-distribution in an array of parallel multiple channels still requires a solution due to poor cooling performance from the non-uniform temperature distribution on the surfaces of heat sinks. A number of experimental works have been conduced to improve flow distribution through header modifications such as the works of Samson et al. (1987), and Hrnjak (2004), or through modification of the channel core, using cross-links (Jiang et al., 2002; Cho et al., 2003; Dang and Hassan., 2007). Such experimental works is an expensive endeavor to seek appropriate designs for microchannel heat sinks. With an advantage of both hardware and software today, CFD (computational fluid dynamics) can be used to pursue the same goal but less expensive effort.

Compared to experimental two-phase studies, lacking data of two-phase characteristics can be found in numerical works at this time due to the complexity of two-phase flow phenomena. A number of works has been numerically investigated twophase flow in single channel, tube bend, T-junction, etc., by using different methods such as VOF (Volume of Fluid), Eulerian-Eulerian, the Level Set, etc. (Qian and Lawal 2006; Yang et al., 2002; Bretherton, 1961; Aliabadi et al. 2002; Conde et al., 2004; Shepel and Smith, 2006; Amornkul et al., 2005; Lun et al., 1996). From these works, method of twophase models can be appropriately used for two-phase flow simulation, is depending on the interest of research field and computer resources available. Numerical investigations of twophase flow in parallel multiple channels are rare in the literature. Some can be found in single phase flow to optimize the geometrical design of parallel multi-channel configurations of heat sinks (Tonomura et al., 2004; Lu and Wang, 2006). Unlike single phase flow, two-phase flow behavior in an array of parallel channel is very complex. Thus, simulation of twophase flow in multiple channels should be expected to cause some issues because of time consumption for convergence, or insufficient memory of available computer resources. Due to such possible problems, computational domains as well as simulation methods are being carefully considered. Moreover, since the universal two phase model is not yet available, various two phase models are still being developed.

\section{METHODS OF MODELING THE EXPERIMENTS}

Three test models, having the same geometry of the test sections in the work of Dang and Hassan (2007), are simulated in the present work. The difference between the three test models is that the cross-links were introduced to the channel core of the standard straight channels model (STR). The cross- linked models, namely CR2 and CR2A, consist of two crosslinks that were located at $1 / 3$ and $2 / 3$ of the channel length, their width varied by one and two times larger the channel width. The computational domain is shown in Figure 1. The origin is located at the center of the bottom surface of the inlet hole. The computational results of three models are validated by comparing them with those from experimental data. Such results then lead to the proposal of a new design, which was also simulated and shown in Figure 2. The difference between the proposed design and the test models is the geometry of cross-links which are inclined to the channels.

The commercial CFD code, Fluent 6.3 , is used to simulate the 3D two-phase flow for a same range of experimental flow conditions, as seen in Table 1. The two-phase flow is reasonably considered as incompressible for a studied flow range. The steady state is also assumed in this study, while the turbulent flow model is taken into accounted. The mixture model incorporated with the standard k- $\varepsilon$ turbulent model, is used to simulate the three dimensional two-phase flows in multiple parallel channels in the present work. The mixture model is a simplified multiphase flow model, and it allows phases to move with different velocities. It is also used as a homogeneous two-phase model, when phases move at the same velocity. The mixture model is therefore used as a suitable twophase model to seek an appropriate design for microchannel heat sinks in terms of flow distribution.

For the inlet boundary condition, the two-phase flow mixture is assumed as homogeneous flow. Moreover, the above assumptions lead to determining the volume fraction and the mixture velocity for the inlet flow boundary condition from the Equations 1 and 2. From the Equation 1, the volume fraction is determined as same as the volume quality, which is the ratio of the volume flow rates of a gas and a mixture. They are therefore considered as the same based on the assumptions stated in this study. The boundary conditions at the channel outlets are set as a pressure outlet, with absolute gauge pressure.

$$
\begin{gathered}
\beta_{G}=\frac{Q_{G}}{Q_{m}} \\
j_{\text {mix }}=\frac{j_{G}}{\beta_{G}}
\end{gathered}
$$

\section{RESULTS AND DISCUSSION}

\section{Mesh Independence and Validation}

Mesh independence is studied for the STR model, using four mesh systems, namely coarse mesh, base mesh, fine mesh and 
finer mesh (Table 2). The structured mesh is used for the header, the channels and the cross-links, whereas the unstructured mesh is used for the inlet tube. The V-velocity profiles at the channel outlets are tested for mesh independence, shown in Figure 3. The results showed that almost the same velocity profiles are observed when using the fine and the finer mesh systems. It is interesting to note that the finer mesh has almost double the number of cells as the fine mesh system. To conserve computational time, the fine mesh was used. This selected mesh type was also used in the remaining cases. The results converge in a range of 3500 to 4500 iterations with $10^{-6}$ residuals.

To validate the results generated from the present work, the mass flow rates of water in four selected channels, 1, 2, 3 and 4 , corresponding to the $1^{\text {st }}$, the $15^{\text {th }}$, the $30^{\text {th }}$, and the $45^{\text {th }}$ channels of 45 channels (Figure 1), are compared with experiment data, as shown in Figure 4, which shows flow comparison for the CR-2A test model. The results show that both numerical and experimental data has the same trend of flow distribution in the four selected channels. Such results were observed for the other cases as well. The results however show that the present data under-predicts the experimental data for all studied cases. It is also observed that flow distribution is fairly symmetrical from numerical results, whereas it is not from the experiment. The discrepancy between the numerical and experimental results is attributed to the CFD assumptions of steady state and homogenous inlet boundary conditions, and as well to the experimental uncertainty.

\section{Flow Distribution}

A CFD work enables to gain easily further insight into flow distribution throughout 45 channels, whereas it is difficult from experiment. Figure 5 presents the sample of flow distribution comparison between the test models at the channel outlets, from Case 3. The results of flow distribution show that high flow occurs at some central channels and some outermost channels for all test models. The results showed symmetrical flow distribution is observed throughout 45 channels for all test models. For simplification, a flow distribution profile can be divided into three regions with respect to the ideal flow distribution. The highest flow region is from the $21^{\text {st }}$ to $23^{\text {rd }}$ channels, the high flow region is from the $1^{\text {st }}$ to channel $9^{\text {th }}$ channels, and the low flow region is from the $10^{\text {th }}$ to $20^{\text {th }}$ channels (Figure 2). These flow regions are considered to be the same as flow distribution in the other side $\left(24^{\text {th }}\right.$ to $45^{\text {th }}$ channels), due to symmetrical flow distribution. In the low flow region, mass flow rate is observed lower than ideal mass flow rate for all test models. However, the deviation between the measured flow rate and the ideal flow rate tends to decrease close to the ideal line, when the cross-links are incorporated. It is also observed in the same results from other flow regions. The lowest standard deviation (Eq. 3) is observed at Case 3. It is good to note that Dang and Hassan (2007) observed intermittent flow patterns (plug and elongated bubble flow patterns) as dominant flow patterns in the straight and the cross-linked models at this case. However, more data points between these studied cases should be investigated to see what flow conditions will result in uniform flow distribution. Compared to the standard straight model (STR), the crosslinked and the proposed models have lower standard deviations in which the standard deviations are improved up to $25 \%, 40 \%$, and $55 \%$ for the CR-2, the CR-2A, and the proposed model, respectively, in the studied cases.

$$
\boldsymbol{S T D E} V=\sqrt{\frac{1}{N-1} \sum_{i=1}^{N}\left(x_{i}-\bar{x}\right)^{2}}
$$

Introducing cross-links into channel core allow flow sharing from high flow channels to low flow channels (Dang and Hassan, 2007). This can be seen from this numerical work in Figure 6, which presents velocity vector of flow field in crosslinks for Case 1. The two-phase mixture velocities show that they traverse from high flow channels to low flow channels along the cross-links. This can be also experimentally observed from the images taken by Dang and Hassan (2007) (Figure 6a). Although the bubbles cannot be seen in the CFD results, it can be shown that the circulation is present in the cross-links and the channels. It is observed that the larger cross-links permit more flow sharing compared to smaller cross-links as seen in Figures $6 \mathrm{a}$ and $\mathrm{b}$. The cross-links are considered as the additional inlets and exits along channels, hence, circulations are also observed in the cross-links between channels, as well as in the channels after flow goes through the cross-links as discussed earlier. This leads to prevent parts of flow sharing among channels through the cross-links. Figure $6 \mathrm{~b}$ also presents the volume fraction of air in the channels. This figure shows that some regions having high volume fraction of air among the channels and the cross-links, for example a value of 0.032 , can mean the possibility of bubbles. For the inclined cross-links, more flow sharing between channels and less circulations were observed. As a result, the proposed design improves flow sharing from high flow channels to low flow channels.

The effect of the cross-links on flow distribution will be more understood from flow analysis through the Figure 7 which presents the sample of $\mathrm{V}$-velocity distribution along the center lines of channels $23^{\text {rd }}$ at Case 1 . The results of velocity distribution in this channel, which is highest flow channel, showed significant improvement for the cross-linked models when compared to the straight model (Figure 5). Moreover, the proposed model shows better improvement. This improvement is observed as the $\mathrm{V}$ - velocity decreases $24.4 \%$ in this channel for the proposed model and $12.6 \%$ and $19.3 \%$ for the CR2 and the CR2A models respectively. The results in overall show how flow distribution can be improved due to the effects of cross-links, and the inclined cross-link geometry is most 
effective in term of flow distribution in the examined range in this study.

\section{Pressure Drop}

Two-phase pressure drop is calculated using the surface weight average from bottom surface of inlet hole (Figure 1), and the results were compared for the test models. The results showed that no significant differences are observed between the test models. Compared to experimental results, the results showed that the present data over-predicts the experimental pressure drop at Case 1, and under-predicts from Cases 2 - 4 for all three test models. The largest average deviation is observed as $65.3 \%$ for the two-phase pressure drop comparison of the STR model, whereas the lowest case is $42.3 \%$ for the CR2A model. However it is good to note that the present data has low deviation from Case 2 to Case 4. As a result, it can fairly predict the experimental pressure drop for the CR2A model from Cases 2 to Case4, with an average deviation of $19.5 \%$.

The pressure drop of the STR model is compared to those obtained from three widely used two-phase pressure drop models. Figure 8 presents the comparison of the present data to the two-phase pressure drop models, the homogeneous model, the Friedel (1979) model, and the Chisholm (1967) model. Similar to the results compared to experimental results, the present data over-predicts two-phase pressure drop from three above models at Case 1. It is interesting to note that the present data showed good agreement with the Friedel model from Case 2 to Case 4 . In these cases, the Friedel model fairly predicts the experimental data, with an average deviation of $28.7 \%$. However, more data should be numerically investigated to verify this agreement.

\section{CONCLUSIONS}

Two-phase flow in microchannel heat sinks is modeled and simulated in the present work under adiabatic condition. The mixture and standard $\mathrm{k}-\varepsilon$ turbulent flow models are used to simulate the three dimensional two-phase flow in three test geometries, namely the STR, the CR2, and the CR2A. Mesh independence is accounted and the suitable structure mesh is used for the two-phase simulation.

The results of flow distribution were validated with experimental results, and the same trend of flow distribution was observed for experimental and numerical results. The new design was proposed and simulated with the three test models in this work. The results showed that the proposed design improves two-phase flow distribution, up to $55 \%$ compared to the standard straight channel model. There are no significant differences between the test models when compared two-phase pressure drop. The numerical two-phase pressure drop overpredicts the experimental results for Case 1. However, the results from the $\mathrm{CR}-2 \mathrm{~A}$ test model fairly predict the experimental results of Case 2 and Case 4. Compared to twophase pressure drop models, the Friedel model fairly predicts the numerical pressure drop for the STR test model from Case 2 to Case 4, while the homogeneous model and the Chisholm model, under-predict the present data for all studied cases.

Although the accuracy of simulation 3D two-phase flow using the CFD code, Fluent, needs further investigations, the results from the present works provide significant data for seeking appropriate designs of parallel multi-channel heat sinks in terms of flow distribution.

\section{FUTURE WORK}

More cross-links, with same geometry of the proposed design in this work, should be investigated while rounding all sharp corners at the channel inlets to lessen the entrance effect. Moreover, various angles between the cross-links and the channels can be taken into account as well as locations of cross-links along channels. Further investigation should be possibly carried out under to-scale conditions for microchannel heat sinks.

\section{REFERENCES}

Aliabadi, S., Johnson, A., Zellars, B., Abatan, A., and Berger, C., "Parallel simulation of flows in open channels", Future Generation Computer System $18,627-637,2002$.

Amornkul, S. S., Steward, F. R., and Lister, D. H., "Modeling two-phase flow in pipe bends", Journal of Pressure Vessel Technology, 127, 204-209, 2005.

Bretherton, F.P., "The motion of long bubbles in tubes", Journal of Fluid Mechanics 10, 166-188, 1961.

Chisholm, D., "A theoretical basis for the Lockhart Martinelli Correlation for two-phase flow", Int. J. Heat Mass Transfer 10, pp. 1767-1778, 1967.

Cho, E. S., Koo, J., Jiang, L., Prasher, R. S., Kim, M. S., Santiago, J. G., Kenny, T. W., and Goodson, K. E., " Experimental study on two-phase heat transfer in microchannel heat sinks with hotspots," Annual IEEE Semiconductor Thermal Measurement and Management Symposium, 242-246, 2003 .

Conde, R., Parra, M. T., Castro, F., Villafruela, J. M., Rodriguez, M. A., and Méndez, C., " Numerical model for two-phase solidification problem in a pipe”, Applied Thermal Engineering 24, 2501-2509, 2004.

Dang, M. and Hassan, I., "The Effects of Cross Links on Adiabatic Two-Phase Flow Characteristics in Scaled Microchannel Heat Sinks " ASME Fifth International Conference on Nanochannels, Microchannels and Minichannels, 2007.

Friedel, L., "Improved friction pressure drop correlations for horizontal and vertical two- phase pipe flow", Paper E2, European Two-Phase Group Meeting, Ispra, Italy, 1979.

Hrnjak, P., "Developing adiabatic two-phase flow in headers- distribution issue in parallel flow microchannels heat exchnahers" Heat Transfer Engineering, 25(3), 63-68, 2004. 
Jiang, L., Koo, J. M., Wang, E., Bari, A., Cho, E. S., Ong, W., Prasher, R. S. Maveety, J., Kim, M. S., Kenny, T. W., Santiago, J. G., and Goodson, K. E., "Cross-linked microchannels for VLSI hotspot cooling. Proc" ASME International Mechanical Engineering Congress \& Exposition, 13-17, 2002.

Lockhart, R.W. and Martinelli, R.G.,. "Proposed correlations for isothermal two-phase two-component flow in pipes" Chem. Eng. Prog. 45, 39-48, 1949.

Lu, M. C. and Wang, C. C., " Effect of the inlet location on the performance of parallel- channel cold - plate", IEEE Transactions on Components and Packing Technologies, 29, 30-38, 2006.

Lun, I., Calay, R. K., and Holdo, A. E., "Modelling two-phase flows using CFD”, Applied Energy, 53 (1996), 299-314.

Maharudrayya, S., Jayanti, and S., Deshpande, A. P., "Pressure drop and flow distribution in multiple parallel-channel configurations used in protonexchange membrane fuel cell stacks", Journal of Power Sources, 157, 358-367, 2006.

Qian, D. and Lawal A., " Numerical study on gas and liquid slugs for Taylor flow in a T-junction microchannel”, Chemical Engineering Science, 61, 76097625,2006

Samson, E., B., Stark, J., A., and Grote, M. G., "Two-phase flow header tests" 17th Intersociety Conference on Environmental Systems, SAE Technical Paper Serie, $17 \mathrm{p}, 1987$.

Shepel, S. V. and Smith, B. L., " New finite-element/finite-volume level set formulation for modeling two-phase incompressible flows", Journal of Computational Physics, 218, 497-494, 2006.

Tuckerman, D. B., and Pease, R. F. W., "High-performance heat sinking for VLSI” IEEE Electron Device Letters, EDL-2, 126-129, 1981.

Tonomura, O., Tanaka, S., Noda, M., Kano, M., Hasebe, S., and Hashimoto, I., "CFD-based optimal design of manifold in plate-fin microdevices", Chemical Engineering Journal, 101, 397-402, 2004.

Yang, Z. L., Palm, B., and Sehgal, B. R., "Numerical simulation of bubbly two-phase flow in a narrow channel", International Journal of Heat and Mass Transfer, 45, 631-639, 2002.

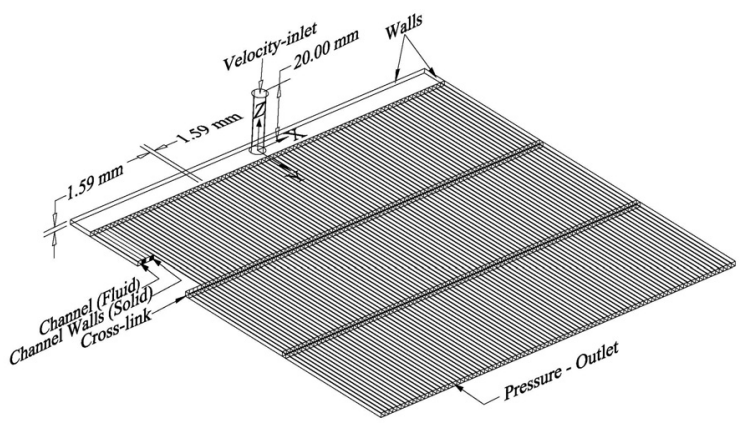

Figure 1. Computational domain.

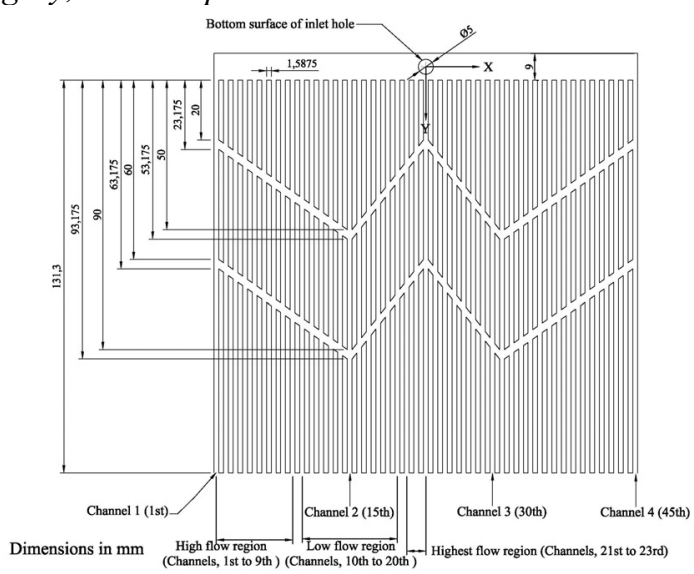

Figure 2. Proposed design

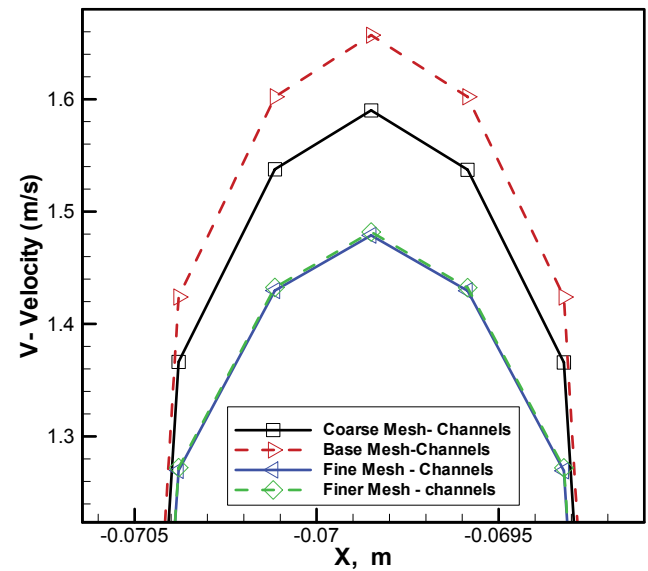

Figure 3. Results of mesh independence test, from the $1^{\text {st }}$ channel.

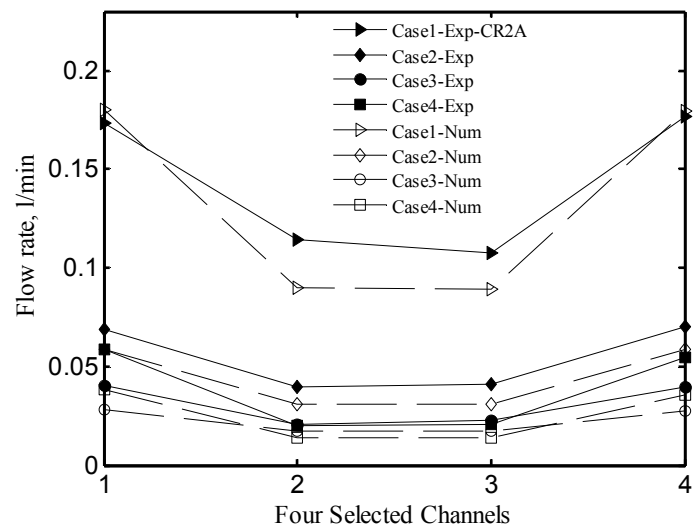

Figure 4. Flow distribution compared to experimental results (CR-2A). 


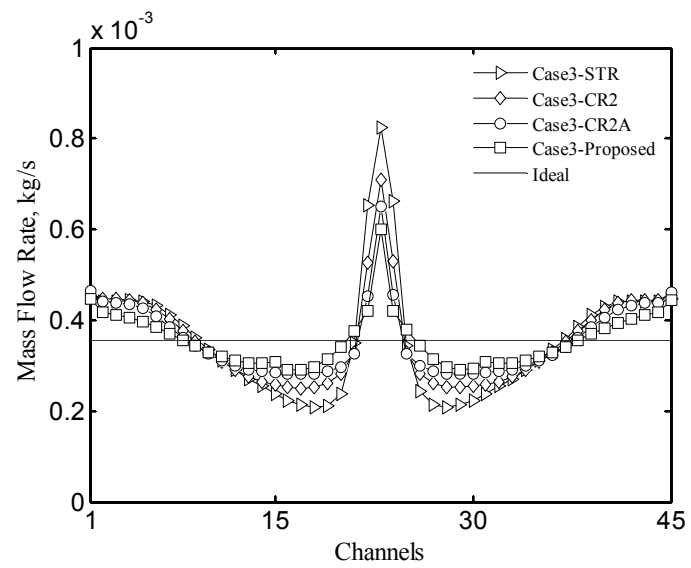

Figure 5. Comparison of flow distribution for all test models, Case 3 ( $\beta=$ $0.7274)$.
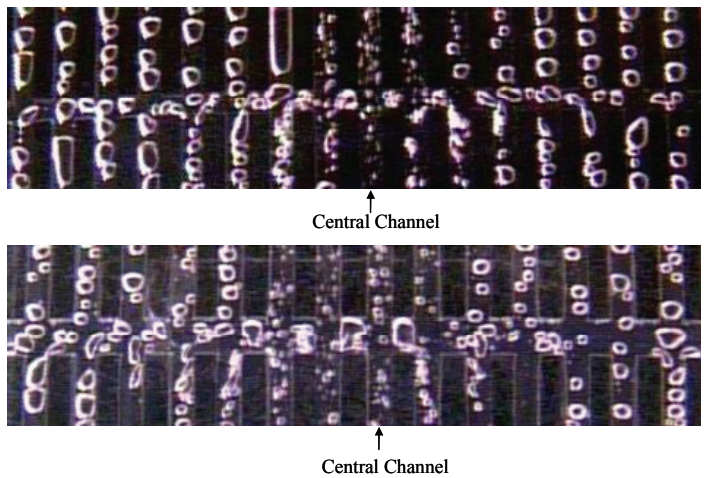

a) Samples of flow interaction- i) CR2; ii) CR2A, from experiment, images taken from Case 1 (Dang and Hassan, 2007).

(Direction of flow is from top to bottom)

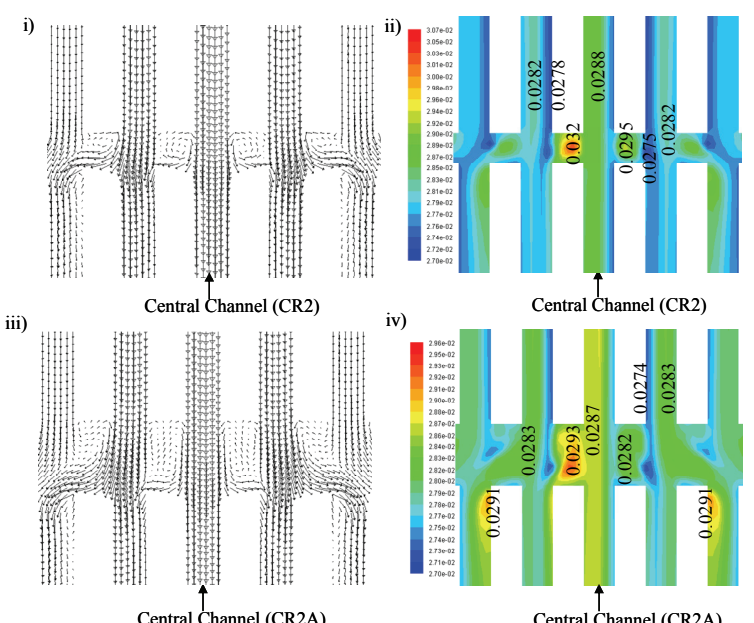

b) Samples of mixture velocity and volume fraction along the cross-links, images taken from Case $1(\beta=0.0286)$.

Figure 6. Sample of flow sharing from cross-links

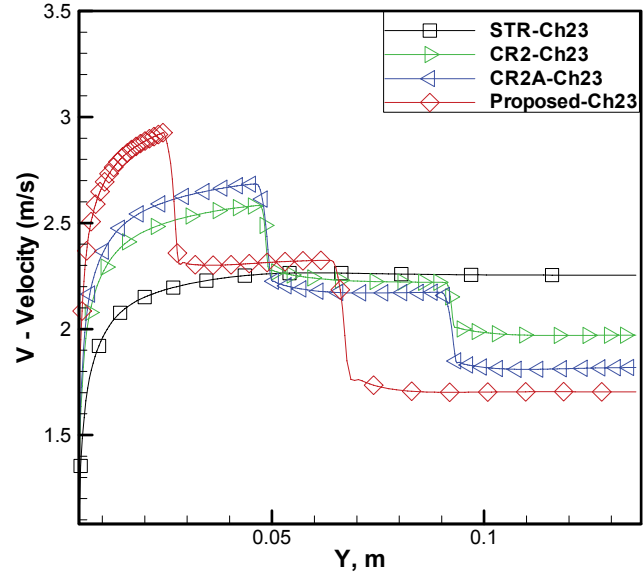

Figure 7. V-velocity profile of the center line in $23^{\text {rd }}$ channel.

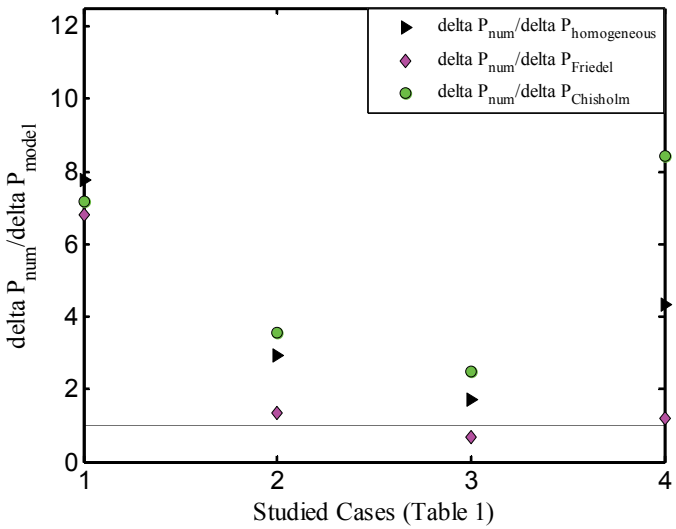

Figure 8. Pressure drop comparison with models, from the STR model.

TABLE 1

EXAMINED FLOW CONDITIONS

\begin{tabular}{|l|l|l|l|l|l|}
\hline & $J_{G}(\mathrm{~m} / \mathrm{s})$ & $J_{L}(\mathrm{~m} / \mathrm{s})$ & $J_{m}(\mathrm{~m} / \mathrm{s})$ & $\beta_{\text {air }}$ & $\mathrm{x}$ \\
\hline 1 & 0.142 & 4.8282 & 4.9702 & 0.0286 & $3 \mathrm{E}-05$ \\
\hline 2 & 2.1855 & 1.5809 & 3.7663 & 0.5803 & 0.0016 \\
\hline 3 & 2.1855 & 0.8189 & 3.0044 & 0.7274 & 0.0032 \\
\hline 4 & 26.642 & 0.8189 & 27.461 & 0.9702 & 0.0371 \\
\hline
\end{tabular}

TABLE 2

MESH INFORMATION

\begin{tabular}{|l|l|}
\hline Examined Mesh & Total number of cells \\
\hline Coarse & 419235 \\
\hline Base & 430329 \\
\hline Fine & 636838 \\
\hline Finer & 1250419 \\
\hline
\end{tabular}

